\title{
THE DENSITY OF PEAK POINTS IN THE SHILOV BOUNDARY OF A BANACH FUNCTION ALGEBRA
}

\author{
TAHER G. HONARY
}

(Communicated by John B. Conway)

\begin{abstract}
H. G. Dales has proved in [1] that if $A$ is a Banach function algebra on a compact metrizable space $X$, then $\bar{S}_{0}(A, X)=\Gamma(A, X)$, where $S_{0}(A, X)$ is the set of peak points of $A$ (w.r.t. $\left.X\right)$ and $\Gamma(A, X)$ is the Shilov boundary of $A$ (w.r.t. $X$ ). Here, by considering the relation between peak sets and peak points of a Banach function algebra $A$ and its uniform closure $\bar{A}$, we present an elementary and constructive proof of the density of peak points in the Shilov boundary.
\end{abstract}

Introduction. For the notations, definitions, elementary and known results, one can refer to [2].

Let $X$ be a compact Hausdorff space and let $C(X)$ denote the space of all continuous complex valued functions on $X$. A function algebra on $X$ is a subalgebra of $C(X)$ which contains the constants and separates the points of $X$. Now consider a norm for the function algebra $A$ on $X$ such that $\|f \cdot g\| \leq\|f\| \cdot\|g\|$ for all $f, g \in A$. A complete normed function algebra on $X$ is called a Banach function algebra on $X$. If the norm of a Banach function algebra is the uniform norm on $X$, i.e., if $\|f\|_{X}=\sup _{x \in X}|f(x)|$, it is called a uniform algebra on $X$. If $A$ is a function algebra on $X$, then $\bar{A}$, the uniform closure of $A$, is a uniform algebra on $X$.

If $(A,\|\cdot\|)$ is a Banach function algebra on $X$, for every $x \in X$ the map $\phi_{x}: A \rightarrow$ C, defined by $\phi_{x}(f)=f(x)$, is a nonzero continuous complex homomorphism on $A$ and so $\phi_{x} \in M_{A}$, where $M_{A}$ is the maximal ideal space of $A$. Clearly for every $f \in A$,

$$
\|f\|_{X}=\sup _{x \in X}|f(x)|=\sup _{x \in X}\left|\phi_{x}(f)\right| \leq \sup _{\phi \in M_{A}}|\phi(f)| \leq\|f\| .
$$

Let $A$ be a function algebra on $X$. A closed subset $P$ of $X$ is called a peak set of $A$ (w.r.t. $X$ ) if there exists a function $f \in A$ such that $f=1$ on $P$ and $|f|<1$ on $X \backslash P$. If $P=\{p\}$, then $p$ is called a peak point of $A$ (w.r.t. $X$ ) and the set of all peak points of $A$ (w.r.t. $X)$ is denoted by $S_{0}(A, X)$. A subset $E$ of $X$ is called a boundary for $A$ (w.r.t. $X$ ) if every function $f \in A$ attains its maximum modulus on $E$. Evidently every boundary must contain $S_{0}(A, X)$. The Shilov boundary of $A$ (w.r.t. $X$ ) is the smallest closed boundary of $A$ (w.r.t. $X$ ) and it is denoted by $\Gamma(A, X)$. It is well known that the Shilov boundary for function algebras exists and it is in fact the intersection of all closed boundaries.

A local characterization of the points of $\Gamma(A, X)$ is clear: A point $x \in X$ is in $\Gamma(A, X)$ if and only if for any neighbourhood $N_{x}$ of $x$ there exists $f \in A$ such that

Received by the editors February 6, 1987 and, in revised form, May 6, 1987.

1980 Mathematics Subject Classification (1985 Revision). Primary 46J10, 46J20. 
the set on which $f$ attains its maximum modulus is contained in $N_{x}$, or, in other words, there exists a peak set $P$ of $A$ such that $P \subseteq N_{x}$.

It is a well-known theorem of Bishop that for a uniform algebra $A$ on a compact metrizable space $X$ every peak set of $A$ contains a peak point of $A$ and so the set of peak points of $A$ is a boundary and therefore is dense in the Shilov boundary of $A$, i.e., $\bar{S}_{0}(A, X)=\Gamma(A, X)$.

H. G. Dales has extended this result in [1] to any Banach function algebra $A$ on a compact metrizable space $X$. Now we give another proof of the density of peak points in the Shilov boundary of a Banach function algebra which is constructive and elementary.

THEOREM. If $A$ is a Banach function algebra on a compact metrizable space $X$, then the set of peak points of $A$ is dense in the Shilov boundary of $A$, i.e., $\bar{S}_{0}(A, X)=\Gamma(A, X)$.

PROOF. It is sufficient to show that any neighbourhood $N_{0}$ of a peak set $P_{0}$ of $A$ contains a peak point of $A$.

If $P_{0}$ is a peak set of $A$ it is also a peak set of $\bar{A}$, and so it contains a peak point $p_{1}$ of $\bar{A}$. Thus there exists $f_{1} \in \bar{A}$ such that $f_{1}\left(p_{1}\right)=1$ and $\left|f_{1}\right|<1$ on $X \backslash\left\{p_{1}\right\}$. Now let $N_{1}$ be a neighbourhood of $p_{1}$ such that $\bar{N}_{1} \subseteq N_{0}$. Take $\beta_{1}=\max _{x \in X \backslash N_{1}}\left|f_{1}(x)\right|$. Then $\beta_{1}<1$. Given $\varepsilon_{1}>0$ there exists $g_{1} \in A$ such that $\left\|g_{1}-f_{1}\right\|_{X}<\varepsilon_{1}$ and so $\left|g_{1}\left(p_{1}\right)\right|-\left|g_{1}(q)\right|>1-2 \varepsilon_{1}-\beta_{1}$ for $q \in X \backslash N_{1}$. By taking $\varepsilon_{1}$ small enough so that $1-2 \varepsilon_{1}-\beta_{1}>0$ we have $\left|g_{1}(q)\right|<\left|g_{1}\left(p_{1}\right)\right|$ for $q \in X \backslash N_{1}$. Thus $g_{1}$ attains its maximum modulus within $N_{1}$.

If $g_{0} \in A$ peaks on $P_{0}$, then

$$
\left|g_{0}\right|+\eta_{1}\left|g_{1}\right|<1+\eta_{1}\left(\varepsilon_{1}+\beta_{1}\right) \quad \text { on } X \backslash N_{1}
$$

and

$$
\left|g_{0}\left(p_{1}\right)\right|+\eta_{1}\left(g_{1}\left(p_{1}\right) \mid>1+\eta_{1}\left(1-\varepsilon_{1}\right) \text { for every } \eta_{1}>0\right.
$$

Hence

$$
\left|g_{0}\left(p_{1}\right)\right|+\eta_{1}\left|g_{1}\left(p_{1}\right)\right|-\left|g_{0}\right|-\eta_{1}\left|g_{1}\right|>\eta_{1}\left(1-2 \varepsilon_{1}-\beta_{1}\right)>0 \quad \text { on } X \backslash N_{1} .
$$

Thus $\left|g_{0}\right|+\eta_{1}\left|g_{1}\right|$ attains its maximum within $N_{1}$. Clearly we can find $\phi_{1}\left(0 \leq \phi_{1} \leq\right.$ $2 \pi$ ) such that $G_{1}=g_{0}+\eta_{1} e^{i \phi_{1}} g_{1}$ attains its maximum modulus within $N_{1}$. In fact

$$
\left\|G_{1}\right\|_{X}-\left|G_{1}(q)\right|>\eta_{1}\left(1-2 \varepsilon_{1}-\beta_{1}\right)>0 \quad \text { for } q \in X \backslash N_{1} .
$$

Since $G_{1} \in A$, the maximum set of $G_{1}$ contains a peak point $p_{2} \in N_{1}$ of $\bar{A}$ and so there exists $f_{2} \in \bar{A}$ such that $f_{2}\left(p_{2}\right)=1$ and $\left|f_{2}\right|<1$ on $X \backslash\left\{p_{2}\right\}$. As before let $N_{2}$ be a neighbourhood of $p_{2}$ such that $\bar{N}_{2} \subset N_{1}$ and take $\beta_{2}=\max _{x \in X \backslash N_{2}}\left|f_{2}(x)\right|$ so that $\beta_{2}<1$. For $\varepsilon_{2}>0$ small enough, there exists $g_{2} \in A$ which attains its maximum modulus within $N_{2}$. Given $\eta_{2}>0$, as before we can construct $G_{2} \in A$ such that $G_{2}=g_{0}+\eta_{1} e^{i \phi_{1}} g_{1}+\eta_{2} e^{i \phi_{2}} g_{2}$ attains its maximum modulus within $N_{2}$ and

$$
\left\|G_{2}\right\|_{X}-\left|G_{2}(q)\right|>\eta_{2}\left(1-2 \varepsilon_{2}-\beta_{2}\right)>0 \text { for } q \in X \backslash N_{2}
$$

Now we continue in this way to get a sequence of neighbourhoods $\left\{N_{n}\right\}_{1}^{\infty}$ such that $\bar{N}_{n} \subseteq N_{n-1}(n=1,2, \ldots)$ and

$$
G_{n}=g_{0}+\eta_{1} e^{i \phi_{1}} g_{1}+\cdots+\eta_{n} e^{i \phi_{n}} g_{n} \in A
$$


attains its maximum modulus within $N_{n}$. Moreover for each $n$,

$$
\left\|G_{n}\right\|_{X}-\left|G_{n}(q)\right|>\eta_{n}\left(1-2 \varepsilon_{n}-\beta_{n}\right)>0 \text { for } q \in X \backslash N_{n}
$$

and so $\left\|G_{n}\right\|_{X} \geq \sup _{q \in X \backslash N_{n}}\left|G_{n}(q)\right|+\eta_{n}\left(1-2 \varepsilon_{n}-\beta_{n}\right)$.

Next take $\Delta_{n}=\left\|G_{n}\right\|_{X}-\sup _{q \in X \backslash N_{n}}\left|G_{n}(q)\right|$ and define the sequence $\left\{\delta_{n}\right\}_{1}^{\infty}$ by $\delta_{n}=\min \left\{1 / n, \Delta_{1}, \Delta_{2}, \ldots, \Delta_{n}\right\}$ and $g=g_{0}+\sum_{n=1}^{\infty} \eta_{n} e^{i \phi_{n}} g_{n}$ by choosing $\eta_{n}$ small enough such that this series is convergent in the norm of $A$. To show this let $g=G_{n}+H_{n}$ and suppose $\eta_{1}, \eta_{2}, \ldots, \eta_{k-1}$ has been chosen so that $G_{1}, G_{2}, \ldots, G_{k-1}$ and hence $\delta_{k-1}$ are determined and define $\eta_{k}=\delta_{k-1} / 2^{k}\left\|g_{k}\right\|$. Since $\delta_{k} \leq \delta_{n}$ for $k \geq n$ we have

$$
\left\|H_{n}\right\|_{X} \leq\left\|H_{n}\right\| \leq \sum_{k=n+1}^{\infty} \eta_{k}\left\|g_{k}\right\|=\sum_{k=n+1}^{\infty} \frac{\delta_{k-1}}{2^{k}} \leq \delta_{n} \sum_{k=n+1}^{\infty} \frac{1}{2^{k}}<\frac{\delta_{n}}{2},
$$

but $\delta_{n} \underset{n \rightarrow \infty}{\rightarrow} 0$ and so $\left\|H_{n}\right\| \underset{n \rightarrow \infty}{\rightarrow} 0$, i.e., the series $\sum_{n=1}^{\infty} \eta_{n} e^{i \phi_{n}} g_{n}$ is convergent in norm of $\vec{A}$. Therefore $g \in A$.

Let $p_{n} \in N_{n}(n=1,2, \ldots)$ such that $\left|G_{n}\left(p_{n}\right)\right|=\left\|G_{n}\right\|_{X}$. Then

$$
\left|g\left(p_{n}\right)\right| \geq\left|G_{n}\left(p_{n}\right)\right|-\left|H_{n}\left(p_{n}\right)\right|>\left\|G_{n}\right\|_{X}-\left\|H_{n}\right\|_{X}>\left\|G_{n}\right\|_{X}-\delta_{n} / 2
$$

and so

$$
\begin{aligned}
|g(q)| & \leq\left|G_{n}(q)\right|+\left|H_{n}(q)\right| \leq \sup _{q \in X \backslash N_{n}}\left|G_{n}(q)\right|+\left\|H_{n}\right\|_{X} \\
& <\left\|G_{n}\right\|_{X}-\Delta_{n}+\delta_{n} / 2 \leq\left\|G_{n}\right\|_{X}-\delta_{n} / 2<\left|g\left(p_{n}\right)\right|
\end{aligned}
$$

for $q \in X \backslash N_{n}$. Therefore $g$ attains its maximum modulus within $N_{n}$ for each $n$ and so $M_{g}=\left\{x \in X:\|g\|_{X}=|g(x)|\right\} \subseteq \bigcap_{n=1}^{\infty} N_{n}$.

Since $X$ is compact it has the finite intersection property and so $\bigcap_{n=1}^{\infty} \bar{N}_{n} \neq \varnothing$. If we choose the sequence $\left\{N_{n}\right\}_{1}^{\infty}$ in such a way that the diameter of $N_{n}$ approaches zero, then $\bigcap_{n=1}^{\infty} N_{n}$ must be a singleton and thus a peak point for $A$. This completes the proof of the theorem.

Special thanks are due to the University for Teacher Education in Tehran who supported me financially throughout this work.

\section{REFERENCES}

1. H. G. Dales, Boundaries and peak points of Banach function algebras, Proc. London Math. Soc. 21 (1971), 121-136.

2. C. E. Rickart, The general theory of Banach algebras, Van Nostrand, Princeton, N. J., 1960.

DEPARTMENT OF MATHEMATICS, UNIVERSity FOR TEACHER EDUCATION, TEHRAN 15614 I. R. IRAN 